\title{
RR Lyrae variables in Galactic globular clusters
}

\section{The observational scenario}

\author{
M. Castellani ${ }^{1}$, F. Caputo ${ }^{1}$, and V. Castellani ${ }^{1,2}$ \\ 1 INAF Osservatorio Astronomico di Roma, via Frascati 33, 00040 Monte Porzio Catone, Italy \\ 2 INFN Sezione di Ferrara, via Paradiso 12, 44100 Ferrara, Italy
}

Received 22 April 2003 / Accepted 14 August 2003

\begin{abstract}
In this paper we revisit observational data concerning RR Lyrae stars in Galactic globular clusters, presenting frequency histograms of fundamentalized periods for the 32 clusters having more than 12 pulsators with well recognized period and pulsation mode. One finds that the range of fundamentalized periods covered by the variables in a given cluster remains fairly constant in varying the cluster metallicity all over the metallicity range spanned by the cluster sample, with the only two exceptions given by M 15 and NGC 6441. We conclude that the width in temperature of the RR Lyrae instability strip appears largely independent of the cluster metallicity. At the same time, it appears that the fundamentalized periods are not affected by the predicted variation of pulsators luminosity with metal abundance, indicating the occurrence of a correlated variation in the pulsator mass. We discuss mean periods in a selected sample of statistically significant "RR rich" clusters with no less than 10 RRab and 5 RRc variables. One finds a clear evidence for the well known Oosterhoff dichotomy in the mean period $<P_{\mathrm{ab}}>$ of ab-type variables, together with a similarly clear evidence for a constancy of the mean fundamentalized period $\langle P f\rangle$ in passing from Oosterhoff type II to type I clusters. On this basis, the origin of the Oosterhoff dichotomy is discussed, presenting evidence against a strong dependence of the RR Lyrae luminosity on the metal content. On the contrary, i) the continuity of the mean fundamentalized period, ii) the period frequency histograms in the two prototypes M 3 (type I) and M 15 (type II), iii) the relative abundance of first overtone pulsators, and iv) the observed difference between mean fundamental $<P_{\mathrm{ab}}>$ and fundamentalized periods $\langle P f\rangle$, all agree in suggesting the dominant occurrence of a variation in the pulsation mode in a middle region of the instability strip (the "OR" zone), where variables of Oosterhoff type I and type II clusters are pulsating in the fundamental or first overtone mode, respectively.
\end{abstract}

Key words. stars: variables: RR Lyr - stars: evolution - stars: horizontal-branch

\section{Introduction}

Since the pioneering paper by Oosterhoff (1939), pulsation periods of RR Lyrae variables in Galactic globular clusters have attracted the attention of several astronomers because of some clear evolutionary signatures. Within the restricted sample of clusters with a rich RR Lyrae population one finds a clear difference in the period distribution of the pulsators. In particular, the mean period of the fundamental (RRab) pulsators follows the so called Oosterhoff dichotomy, grouping below (Oosterhoff type I: OoI) or above (Oosterhoff type II: OoII) the value $\left\langle P_{\mathrm{ab}}\right\rangle=0.6$ day, or $\left\langle\log P_{\mathrm{ab}}\right\rangle=-0.22$. There is now a general agreement on the evidence that the dichotomy appears correlated with the cluster metallicity, the more metal poor clusters (OoII) having larger mean periods than moderately metal rich (OoI) ones. However, the origin of such a behavior has raised a long standing debate which remains unsettled.

Send offprint requests to: M. Castellani, e-mail: mkast@mporzio.astro.it
The first pioneering attempt to link stellar evolution to pulsation was given by Rood (1973), whose synthetic Horizontal Branch (SHB) simulations opened the way to a most relevant field of investigation but also revealed, at that time, an irremediable discrepancy between predicted and observed periods. The issue was further investigated by several authors (see, e.g., Caputo et al. 1987; Lee et al. 1990; Caputo et al. 1993; Lee et al. 1994; Demarque et al. 2001) who discussed several important features such as the dependence of the RR Lyrae absolute magnitude $M_{V}(\mathrm{RR})$ on metallicity and HB morphology, the period-shift at fixed effective temperature, and the second parameter that, in addition to metal abundance, influences HB morphology.

More recently, both the stellar evolution and the pulsation theory have greatly improved, whereas no exhaustive investigations have appeared in the relevant literature discussing synthetic period distributions of RR Lyrae stars at various metal content. On this basis, we planned to revisit this problem on modern grounds, presenting in this first paper a discussion of the observational scenario concerning RR Lyrae periods in 

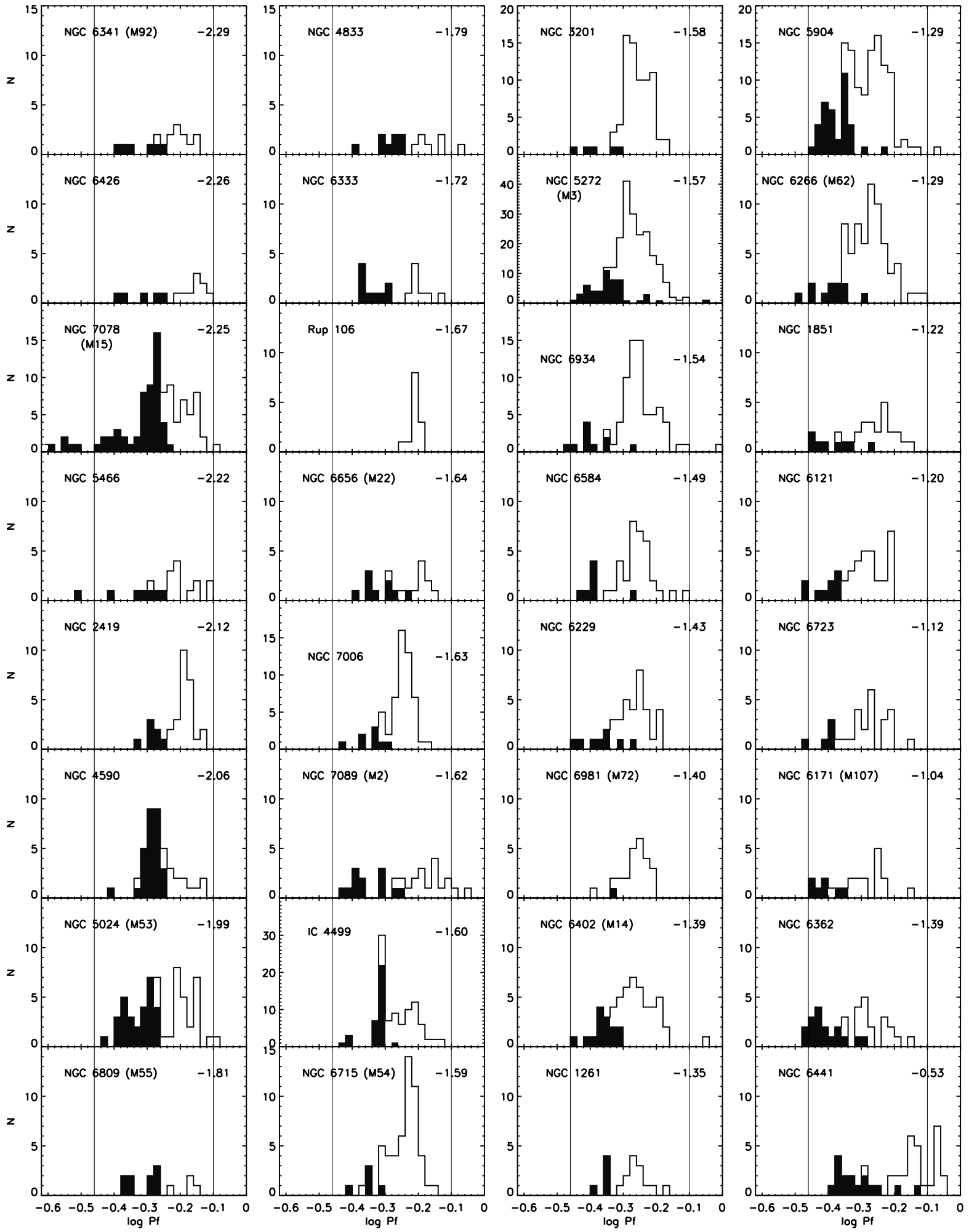

Fig. 1. Period frequency histogram for the selected cluster sample. White and black bars give the contribution of fundamental and fundamentalized first overtone pulsators, respectively.

Galactic globulars. This is intended as a first step in a more extended investigation we are performing on the basis of the predictions from updated pulsation models collated with SHB simulations. The theoretical framework will be presented and discussed in a series of forthcoming papers, together with the analysis of RR Lyrae stars in individual clusters.
In the following, we will take advantage of the careful catalogue of RR Lyrae data made available by Clement (see Clement et al. 2001) to present an up-to-date compilation of period frequency histograms for RR Lyrae stars in a selected sample of Galactic globular clusters. The last collection of these histograms date back to more than fifteen years ago 
(Castellani \& Quarta 1987: hereinafter CQ87) whereas in the meantime observational data have been significantly improved. In the next section we will present these histograms, discussing some relevant evidence to the light of current theoretical predictions. The behavior of mean periods will be investigated in Sect. 3, with particular attention to the sample of clusters with a statistically significant RR Lyrae population. Section 4 will deal with the origin of the Oosterhoff dichotomy, whereas brief remarks will close the paper.

\section{The period frequency histograms}

To enlighten the role of period frequency histograms let us remind that both on theoretical and observational ground we know that within the instability strip one finds either first overtone (RRc) and fundamental (RRab) pulsators, with only first overtone or fundamental mode being unstable at the larger or at the lower temperatures, respectively. Moreover, the period of a RR Lyrae pulsator can be quite firmly linked to the pulsator evolutionary parameters (mass, luminosity and effective temperature), with periods becoming larger when the luminosity increases or the effective temperature and/or the stellar mass decrease. Thus, in each cluster the observed distribution of periods is a signature of the distribution of the mass, luminosity and effective temperature of the pulsators.

As early stated by Stobie (1971), the correct way to investigate such a distribution is to transform the periods so that all the variable are pulsating in one mode, say the fundamental one. According to the pulsation models by van Albada \& Baker (1973), this can be done with good accuracy by simply adopting for c-type pulsators the "fundamentalized" period $\log P f=$ $\log P c+0.13$, as already given in CQ87. More recent computations (Bono et al. 1997, but see also Marconi et al. 2003, Paper II) support such a procedure, with an uncertainty on the fundamentalized periods not larger than $\delta \log P_{\mathrm{f}}= \pm 0.005$. The advantage of using fundamentalized periods is that in this way both RRc and RRab periods follow just the same pulsation relation, so that the observed distribution of fundamentalized periods is directly reflecting the actual distribution of star masses, luminosities and effective temperatures across the whole instability strip.

For our compilation we selected from Clement's catalogue the sample of Galactic globular clusters with more than 12 pulsators with well determined period and pulsation mode, assumed as representative of clusters with a not occasional occurrence of RR Lyrae stars. Presently one finds 32 clusters in this class, against 28 in the previous CQ87 paper, with a total of 1545 variables and an overall increase in the number of pulsators larger than $30 \%$. Figure 1 shows the period frequency histograms for the quoted cluster sample, as arranged in order of increasing metallicity $[\mathrm{Fe} / \mathrm{H}]$ as given in the 1999 update of Harris (1996) compilation. In the figure, the black areas give the contribution of fundamentalized c-type periods. As well known, one finds that first overtone pulsators contribute to the shortest portion of the period histograms, according to their location on the hotter side of the instability strip.

In Fig. 1 one may notice that the range of periods covered by $\mathrm{M} 3$ pulsators can be taken as roughly representative of the behavior of pulsators in the large majority of clusters, independently of the metallicity, with only a minor suggestion for a possible shift toward smaller periods at the larger metallicities. Here we do not give too much relevance to such an evidence, which will be better discussed in forthcoming papers relying on SHB procedures. We only notice that a constant minimum period over the metallicity range $Z=0.0001$ to 0.001 is the one predicted on the basis of HB stellar models, since an increase in metallicity is expected to decrease both luminosity and mass in such a way to keep periods roughly constant, or at the most to decrease periods by $\Delta \log P \sim 0.01$ (see Bono et al. 1995), whereas a slight decrease of period is expected when further increasing the metal content above $Z=0.001$. The above agreement between these predictions and observation supports the theoretical dependence of evolutionary parameters on metallicity.

By looking in Fig. 1 one finds two histograms which clearly fall outside the M 3 period range, as given by M 15 (and perhaps NGC 5466), with the occurrence of a small number of very short period $(\log P f \leq-0.5)$ pulsators, and by NGC 6441, where periods appears clearly shifted toward larger values. As for M 15, it appears hard to account for the shortest periods moving either the star evolutionary parameters or the instability boundaries within reasonable values. However, these pulsators have been found (Butler et al. 1998) in the crowded central cluster region, with only a partial coverage of the light curve. If these periods will be confirmed, this could be regarded as an evidence for second overtone instability, an occurrence not foreseen by current theoretical investigations. As for NGC 6441, a very metal rich cluster with the HB reaching quite hot effective temperatures (Rich et al. 1997), the peculiar large periods of RR Lyrae stars cannot be understood within the current evolutionary scenario (see Sweigart \& Catelan 1998), requiring "ad hoc" assumptions as we will discuss in a forthcoming paper on the basis of synthetic HB simulations. We note that anomalously large RR Lyrae periods have been found also in NGC 6388, another metal rich cluster with peculiarly extended HB (Pritzl et al. 2002). Given the small numbers of well studied variables (4 RRab and $7 \mathrm{RRc}$ ), this cluster is not included in the present study.

\section{Mean periods}

As often adopted in the current literature, the behavior of RR Lyrae in globular clusters can be discussed in terms of mean periods for fundamental and/or first overtone pulsators. However, when approaching such an issue, one has to bear in mind that observational data are affected by statistical fluctuations. In CQ87 we have already approached the problem, showing that typical statistical uncertainties on mean (fundamental or fundamentalized) periods in cluster with 20,40 or 80 RR Lyrae stars are of the order of $\delta \log P \sim \pm 0.03,0.02$ or 0.01 , respectively. Moreover, one has to care about the occurrence of clusters with only a partial population of the strip, where mean periods are governed by this peculiar occurrence. According to these warnings, to discuss mean periods we selected from the sample of 32 clusters those having no less than $10 \mathrm{RRab}$ and $5 \mathrm{RRc}$ with well determined period, fixing 


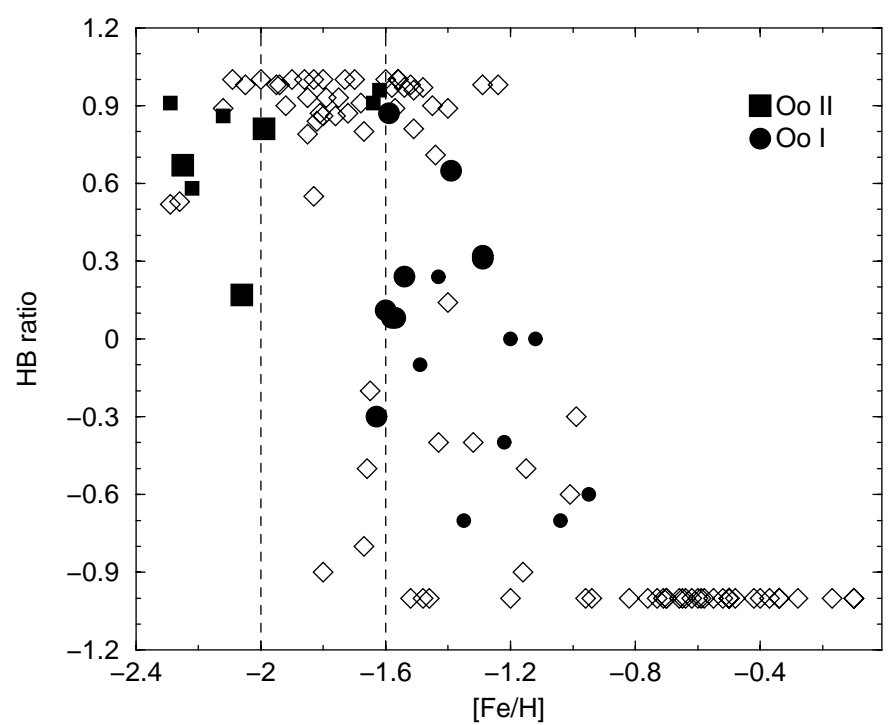

Fig. 2. HB type versus cluster $[\mathrm{Fe} / \mathrm{H}]$ form Harris catalogue. Filled symbols show the sample of RR rich clusters, the largest symbols indicating clusters with more than 40 RR Lyrae variables.

however the attention on clusters with more than 40 RR Lyrae stars from which one expects more stringent indications.

Table 1 ranks these clusters in order of increasing Harris's metallicity, giving for each cluster, left to right, the NGC/IC and (if existing) the Messier number, the iron abundance according to Harris or in the Carretta \& Gratton (1997) scale, the HB type (Lee 1990, from Harris catalogue), and the total number of RR Lyrae stars found in the cluster, followed by the numbers of pulsators recognized as RRab or RRc, including in the latter class the suspected or suggested second overtone pulsators. The following two columns give the mean (logarithmic) period for the fundamental pulsators $\left(<\log P_{\mathrm{ab}}>\right)$ and for the whole sample $(<\log P f>)$, after fundamentalising the RRc periods. Last column finally gives the cluster Oosterhoff type.

The position of this sample of "RR Lyrae rich clusters" within the family of Galactic globular clusters is shown by filled symbols in Fig. 2, where we report the Lee's diagram (HB type against metallicity) for all the globulars with the HB type listed in the Harris catalogue, by adopting Harris metal abundances.

Data in this figure, though affected by observational uncertainties, deserve some comments. Taken at their face value, the data reveal the well known occurrence of a "second" parameter beside $[\mathrm{Fe} / \mathrm{H}]$ governing the $\mathrm{HB}$ morphology, since clusters with metallicity around $[\mathrm{Fe} / \mathrm{H}] \sim-1.4$ may have $\mathrm{HB}$ type ranging from +1 to -1 , i.e., from very blue to completely red HB population. Moreover, as already discussed in CQ87, one finds that the "RR Lyrae rich" clusters (filled symbols) form two separate groups, with no cluster in the metallicity range $[\mathrm{Fe} / \mathrm{H}] \sim-1.6$ to -2.0 .

Inspection of data in Table 1 shows that such a dichotomy is preserved even adopting the alternative Carretta \& Gratton (1997) metallicity scale. Below $[\mathrm{Fe} / \mathrm{H}] \sim-2.0$ one finds only OoII type clusters, whereas above $[\mathrm{Fe} / \mathrm{H}] \sim-1.6$ one finds the whole family of OoI clusters plus two OoII clusters (M 2 and M 22) placed just at the lower metallicity edge.
The occurrence of these two "anomalous" OoII clusters is among the observational evidences to be explained by a theoretical scenario for RR pulsators in globulars. Here we only notice that both clusters are not very rich in variables, presenting a blue $\mathrm{HB}$ population.

Concerning the mean periods, the upper panel in Fig. 3 shows the mean fundamental period $<\log P_{\mathrm{ab}}>$ of OoI (filled dots) and OoII (filled squares) clusters in Table 1, as a function of the cluster metallicity, while the lower panel gives the distribution for the mean fundamentalized period $\langle\log P f>$. One finds that the stringent selection of clusters with more than 40 RR Lyrae stars allows to derive from these well known and well discussed diagrams (see, e.g., Clement et al. 2001) some relevant points. From data in the upper panel, one finds that $<\log P_{\mathrm{ab}}>$ follows reasonably strictly the cluster metallicity, with a clear separation between OoII and OoI types which arrange around $<\log P_{\mathrm{ab}}>\sim-0.20$ and -0.25 , respectively, and with an indication for further decreasing periods at the metal rich tail. One has to notice the sudden dichotomy separating OoII from OoI clusters even for clusters with similar metallicity such the couples M 22-M 2 (OoII) and NGC 7006-IC 4499 (OoI). The peculiar cluster NGC 6441, as marked in Fig. 3 with a triangle, will be not further discussed in this paper.

However, the most interesting feature appears in the lower panel of the figure, where one finds again a correlation between $<\log P f>$ and the cluster metallicity, but with the additional evidence that the mean fundamentalized period is constant for metal content lower that $[\mathrm{Fe} / \mathrm{H}]=-1.6$, so that a common value is "connecting" OoII and OoI clusters across the metallicity gap, and with again the indication for a period decrease at the metal rich tail. As a whole, data in Fig. 3 suggest that $<\log P f>$ in RR Lyrae rich clusters does closely follow the cluster metal content, with the mean periods in RR Lyrae less populated clusters being spread off because of statistical fluctuation and/or strong inhomogeneity in the strip population.

However, following the referee's suggestion, one can explore the behavior of RR Lyrae poor clusters in the "metallicity gap" $-2.0<[\mathrm{Fe} / \mathrm{H}]<-1.6$ between OoI and OoII types, by looking at the cumulative contribution of the known variables in clusters of comparable metal content. Our original intention was to group the clusters according to both their metallicity and HB type. However, one finds that in the quoted metallicity range all the clusters containing well studied RR Lyrae stars, but Ruprecht 106, have blue HB populations, with HB types larger than +0.5 . As for Ruprecht $106([\mathrm{Fe} / \mathrm{H}]=-1.67, \mathrm{HB}$ type -0.82 ), it has repeatedly found to be younger than other galactic globulars (see, e.g., Buonanno et al. 1993) and appears as a peculiar OoI type cluster, with $13 \mathrm{RRab}\left(<\log P_{\mathrm{ab}}>=\right.$ -0.24) and no RRc variables. Excluding this object, the remaining clusters have been collected in two groups according to their metallicity, as shown in Table 2.

It turns out that both groups behave as typical OoII type clusters, with mean $<P_{\mathrm{ab}}>$ and $<P f>$ periods in good agreement with the values found in RR Lyrae rich OoII type clusters. Adding these results (open squares) to the data already plotted in the previous Fig. 3, one finds an increasing evidence for a sudden dichotomy of $<\log P_{\mathrm{ab}}>$ around 
Table 1. Selected quantities for Galactic globular clusters with no less than 10 RRab and 5 RRc. Bold characters with asterisks mark the restricted sample of clusters with more than 40 RR Lyrae stars.

\begin{tabular}{|c|c|c|c|c|c|c|c|c|c|c|}
\hline NGC & Messier & {$[\mathrm{Fe} / \mathrm{H}]_{\mathrm{H}}$} & {$[\mathrm{Fe} / \mathrm{H}]_{\mathrm{CG}}$} & HB type & $\mathrm{RR}$ & RRab & $\mathrm{RRc}$ & $<\log P_{\mathrm{ab}}>$ & $<\log P f>$ & Oo-type \\
\hline NGC 6341 & M 92 & -2.29 & -2.16 & 0.91 & 17 & 11 & 6 & -0.201 & -0.244 & II \\
\hline *NGC 7078 & M 15 & -2.25 & -2.12 & 0.67 & 105 & 39 & 57 & -0.192 & -0.258 & II \\
\hline NGC 5466 & & -2.22 & -2.1 & 0.58 & 22 & 13 & 7 & -0.193 & -0.229 & II \\
\hline NGC 2419 & & -2.12 & -1.97 & 0.86 & 34 & 25 & 7 & -0.185 & -0.207 & II \\
\hline *NGC 4590 & M 68 & -2.06 & -1.99 & 0.17 & 44 & 14 & 28 & -0.211 & -0.264 & II \\
\hline *NGC 5024 & M 53 & -1.99 & -1.81 & 0.81 & 59 & 29 & 29 & -0.190 & -0.260 & II \\
\hline NGC 6656 & M 22 & -1.64 & -1.48 & 0.91 & 26 & 10 & 9 & -0.201 & -0.257 & II \\
\hline *NGC 7006 & & -1.63 & -1.35 & -0.28 & 63 & 50 & 8 & -0.245 & -0.259 & I \\
\hline NGC 7089 & M 2 & -1.62 & -1.48 & 0.96 & 30 & 18 & 12 & -0.168 & -0.241 & II \\
\hline *IC 4499 & & -1.60 & -1.46 & 0.11 & 98 & 63 & 34 & -0.239 & -0.270 & I \\
\hline *NGC 6715 & M 54 & -1.59 & -1.25 & 0.87 & 67 & 50 & 5 & -0.240 & -0.250 & I \\
\hline *NGC 3201 & & -1.58 & -1.23 & 0.08 & 81 & 71 & 5 & -0.256 & -0.264 & I \\
\hline *NGC 5272 & M 3 & -1.57 & -1.34 & 0.08 & 235 & 173 & 51 & -0.254 & -0.274 & I \\
\hline *NGC 6934 & & -1.54 & -1.30 & 0.25 & 79 & 68 & 10 & -0.245 & -0.264 & I \\
\hline NGC 6584 & & -1.49 & -1.30 & -0.15 & 42 & 33 & 7 & -0.254 & -0.276 & I \\
\hline NGC 6229 & & -1.43 & -1.30 & 0.24 & 38 & 30 & 8 & -0.259 & -0.282 & I \\
\hline *NGC 6402 & M 14 & -1.39 & -1.27 & 0.65 & 55 & 40 & 14 & -0.246 & -0.276 & I \\
\hline NGC 1261 & & -1.35 & -1.08 & -0.71 & 19 & 13 & 5 & -0.257 & -0.284 & I \\
\hline *NGC 5904 & M 5 & -1.29 & -1.11 & 0.31 & 132 & 88 & 37 & -0.261 & -0.294 & I \\
\hline *NGC 6266 & M 62 & -1.29 & -1.04 & 0.32 & 74 & 62 & 12 & -0.268 & -0.289 & I \\
\hline NGC 1851 & & -1.22 & -1.05 & -0.36 & 29 & 21 & 8 & -0.246 & -0.284 & I \\
\hline NGC 6121 & M 4 & -1.20 & -1.19 & -0.06 & 39 & 30 & 9 & -0.272 & -0.304 & I \\
\hline NGC 6723 & & -1.12 & -0.96 & -0.08 & 29 & 23 & 5 & -0.270 & -0.294 & I \\
\hline NGC 6171 & M 107 & -1.04 & -0.91 & -0.73 & 22 & 15 & 7 & -0.272 & -0.317 & I \\
\hline NGC 6362 & & -0.95 & -0.96 & -0.58 & 36 & 18 & 18 & -0.265 & -0.333 & I \\
\hline *NGC 6441 & & -0.53 & & 0.00 & 43 & 27 & 16 & -0.128 & -0.191 & $?$ \\
\hline
\end{tabular}

$[\mathrm{Fe} / \mathrm{H}] \sim-1.6$, together with a beautiful support to the constancy of $<\log P f>$ passing from OoII to OoI type clusters.

We have extended the analysis also to the remaining RR Lyrae poor clusters with blue HB morphology (HB type $\geq$ 0.80 ) and even larger metal abundance. As listed in Table 2, this third group characterized by OoI-like metal abundance $(<[\mathrm{Fe} / \mathrm{H}]>\sim-1.45)$ discloses OoII features as the mean $<P_{\mathrm{ab}}>$ period is concerned, providing additional proofs that the Oosterhoff dichotomy is not a matter of metal abundance only, but is also governed by the HB morphology.

\section{The Oosterhoff controversy}

As recalled in the introduction to this paper, since long time we are facing the puzzling evidence for the dichotomized behavior of the mean period of fundamental variables in Galactic globular clusters. As for the origin of such a dichotomy, the larger $<P_{\mathrm{ab}}>$ observed in OoII type clusters could be the direct result of common larger pulsator periods, as suggested by Sandage (1982). However, according to an early hypothesis by van Albada \& Baker (1973), OoII type clusters could have longer mean period $\left\langle P_{\mathrm{ab}}>\right.$ for the simple reason that the low period fundamental pulsators observed in OoI clusters are now pulsating in the first overtone, and thus are not contributing to the average of RRab periods. Further pulsational calculations
(Stellingwerf 1975) supported such a picture, disclosing the occurrence within the instability strip of an "OR" zone, where the stars can pulsate either in the fundamental or in first overtone mode, separating the regions where only the first overtone (at larger effective temperatures: FO region) or only the fundamental mode (at lower effective temperatures: $F$ region) are stable (see also Paper II and references therein).

Based on the evidence presented in the previous sections, we are now in the position to draw some firm conclusions. As a first point, one may notice that the already quoted good constancy of the fundamentalized period range in both OoI and OoII clusters indicates that the width in temperature of the strip remains honestly constant, ruling out in the meantime the possibility that OoII pulsators have simply larger luminosity and, thus, larger periods than OoI pulsators. In that case, given the difference $\Delta<\log P_{\mathrm{ab}}>=0.06$ between the two prototypes M 15 (OoII) and M 3 (OoI), one would indeed expect the whole period histogram of M 15 shifted by the same amount with respect to M 3, and this is not the case. Such a conclusion is reinforced by data plotted in Fig. 3, showing that OoI and OoII clusters at $[\mathrm{Fe} / \mathrm{H}] \leq-1.6$ have quite similar $\langle\log P f>$. This is exactly what expected if the Oosterhoff dichotomy is produced by a change in the pulsation mode, which obviously does not affect the fundamentalized periods. 
Table 2. RR Lyrae poor clusters as grouped according to their metallicity. Bold characters with asterisks gives the cumulative properties of the three groups.

\begin{tabular}{|c|c|c|c|c|c|c|c|c|}
\hline NGC & Messier & {$[\mathrm{Fe} / \mathrm{H}]_{\mathrm{H}}$} & HB type & RRab & $\mathrm{RRc}$ & $<\log P_{\mathrm{ab}}>$ & $<\log P f>$ & Oo-type \\
\hline NGC 4833 & & -1.79 & 0.93 & 7 & 8 & & & \\
\hline NGC 5897 & & -1.80 & 0.86 & 3 & 8 & & & \\
\hline NGC 6809 & M 55 & -1.81 & 0.87 & 4 & 9 & & & \\
\hline NGC 4147 & & -1.83 & 0.55 & 6 & 10 & & & \\
\hline NGC 2298 & & -1.85 & 0.93 & 1 & 3 & & & \\
\hline NGC 5824 & & -1.85 & 0.79 & 8 & 1 & & & \\
\hline *Group 1 & & $-1.82 \pm 0.03$ & $\geq 0.55$ & 29 & 39 & -0.190 & -0.257 & II \\
\hline NGC 1904 & M 79 & -1.57 & 0.89 & 2 & 1 & & & \\
\hline NGC 5986 & & -1.58 & 0.97 & 7 & 1 & & & \\
\hline NGC 5286 & & -1.67 & 0.80 & 9 & 5 & & & \\
\hline NGC 6333 & M 9 & -1.72 & 0.87 & 8 & 9 & & & \\
\hline NGC 6093 & M 80 & -1.75 & 0.93 & 4 & 2 & & & \\
\hline *Group 2 & & $-1.66 \pm 0.09$ & $\geq 0.80$ & 30 & 18 & -0.196 & -0.250 & II \\
\hline NGC 288 & & -1.24 & 0.98 & 1 & 1 & & & \\
\hline NGC 6235 & & -1.40 & 0.89 & 2 & 1 & & & \\
\hline NGC 6626 & M 28 & -1.45 & 0.90 & 8 & 2 & & & \\
\hline NGC 6681 & M 70 & -1.51 & 0.96 & 1 & 1 & & & \\
\hline NGC 7492 & & -1.51 & 0.81 & 1 & 2 & & & \\
\hline NGC 6205 & M 13 & -1.54 & 0.97 & 1 & 4 & & & \\
\hline *Group 3 & & $-1.45 \pm 0.07$ & $\geq 0.81$ & 14 & 11 & -0.215 & -0.271 & II \\
\hline
\end{tabular}

One can find several additional evidences supporting these conclusions. The direct comparison of the period frequency histograms in M 3 and M 15, as given in Fig. 4, discloses the occurrence of an intermediate range of fundamentalized periods where a substantial amount of stars is pulsating as first overtone in $\mathrm{M} \mathrm{15}$, but as fundamental pulsators in M 3. We conclude that the variation in the pulsation mode, even if not the unique cause, does play a dominant role in governing the Oosterhoff dichotomy.

Moreover, an overall picture of the Oosterhoff dichotomy must include the well-known variation in the relative abundance of c-type RR Lyrae stars, as given by the number ratio $N_{\mathrm{c}} / N_{\text {tot }}$, where $N_{\text {tot }}=N_{\mathrm{ab}}+N_{\mathrm{c}}$. As shown in Fig. 5, the most RR Lyrae-rich clusters show indeed that the $\left\langle P_{\mathrm{ab}}\right\rangle$ dichotomy (dashed lines) is accompanied by a bimodality (arrows) in the ratio $N_{\mathrm{c}} / N_{\text {tot }}$ which cannot be ascribed to a simple period variation. On the other side, moving the transition from RRab to RRc variables toward lower temperatures (i.e., transforming fundamental in first overtone pulsators) has the twofold effect of increasing $\left\langle P_{\mathrm{ab}}>\right.$ and increasing the relative number of first overtones. If $\langle\log P f>$ is plotted against $N_{\mathrm{c}} / N_{\text {tot }}$, the dichotomy in periods obviously disappears, while remains the signature of the variation in the transition temperature, as given by the larger amount of first overtone pulsators in OoII type clusters.

One may finally reinforce this conclusion on the basis of simple theoretical considerations. In a uniformly populated instability strip, if - as expected - pulsator luminosities and masses can be assumed as honestly constant, the difference $\Delta \log P=<\log P_{\mathrm{ab}}>-<\log P f>$ depends only on the difference $\log T e(\mathrm{FOBE})-\log T e(\mathrm{TR})$ between the temperature of the First Overtone Blue Edge (FOBE: instability blue boundary) and the transition temperature, independently of the pulsator luminosity. On this basis, the significative variation of $\Delta \log P$ between OoI and OoII clusters appearing in the previous Fig. 4 has to be ascribed to a variation in the difference $\log T e(\mathrm{FOBE})-\log T e(\mathrm{TR})$.

On a quantitative point of view, at constant mass and luminosity, one has $\log P=$ const $-3.36 \log T e$ (Paper II, but see also van Albada \& Baker 1973). It follows

$<\log P_{\mathrm{ab}}>-<\log P f>=1.68[\log T e(\mathrm{FOBE})-\log T e(\mathrm{TR})]$.

Adopting from the most RR Lyrae rich clusters in Fig. 3

$<\log P_{\mathrm{ab}}>-<\log P f>\sim 0.02$ (OoI) or $\sim 0.06$ (OoII)

one derives

$\log T e(\mathrm{FOBE})-\log T e(\mathrm{TR}) \sim 0.01(\mathrm{OoI})$ or 0.04 (OoII)

in reasonable agreement with theoretical predictions concerning the range of temperatures covered by the FO region or the FO plus the OR region, respectively (see Fig. 1 in Bono et al. 1997).

This scenario is supported by numerical experiments based on synthetic HB procedures, allowing to take into account realistic variations in mass and luminosity, as well as the effects of not uniform strip populations. To this purpose we made use of HB models as computed in the case of inefficient element sedimentation and adopting the mixing length parameter $l=2.0 H_{\mathrm{p}}$, as calibrated on globular cluster Red Giant Branches (Cassisi et al. 1999). Figure 6 shows the predicted $\Delta \log P$ and 


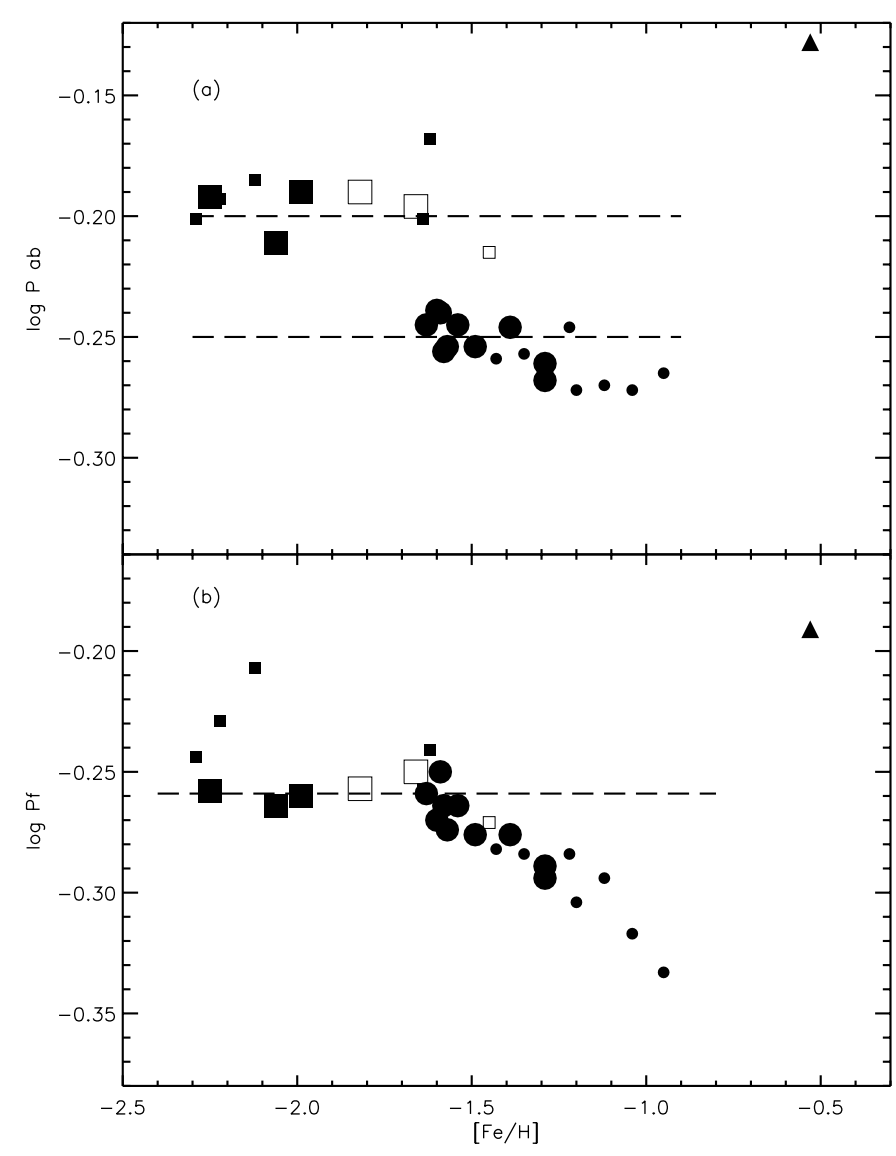

Fig. 3. Mean periods of fundamental a) or fundamentalized b) RR Lyrae pulsators as a function of the cluster metallicity for the sample of 26 "RR rich" globular clusters in Table 1, with filled symbols as in Fig. 2. Open symbols refer to the three selected groups of "RR poor" clusters in Table 2 (see text). The triangle depicts the "anomalous" cluster NGC 6441.

$N_{\mathrm{c}} / N_{\text {tot }}$ ratios inferred by synthetic simulations with HB type in the range of $\sim-0.75$ to $\sim+0.75$, as evaluated under selected assumptions about the difference between $T e(\mathrm{FOBE})$ and the transition temperature $(\Delta \log T e=0.01,0.02$, and 0.04$)$. One finds that for each given $\Delta \log T e$, different HB star distributions can move the predicted $\Delta \log P$ and $N_{\mathrm{c}} / N_{\text {tot }}$ ratio only by a limited amount. Conversely, observational data run against a common $\Delta \log T e$, with first overtone pulsators in OoI clusters covering an effective temperature interval of the order of $\log T e(\mathrm{FOBE})-\log T e(\mathrm{TR}) \sim 0.01-0.02$, whereas in OoII clusters they suggest a much larger value, of the order of $\sim 0.04$.

\section{Final remarks}

In this paper we have revisited observational data concerning RR Lyrae pulsators in Galactic globular clusters, presenting period frequency histograms for variables in the 32 clusters having more than 12 RR Lyrae with well recognized period and pulsation mode. Inspection of the histograms shows that the range of fundamentalized periods covered by variables in a cluster remain roughly constant in varying the cluster metallicity all over the cluster sample, with only two exceptions and with a minor suggestion for a possible shift toward smaller

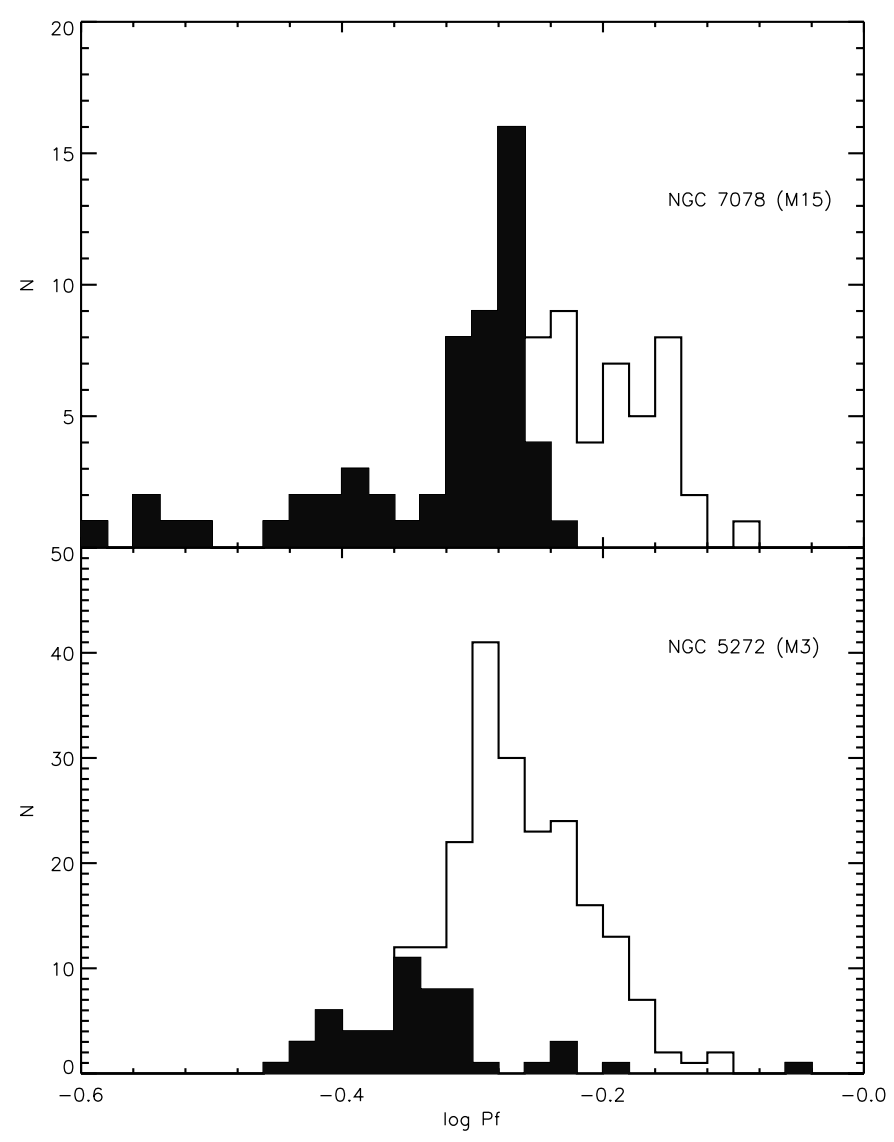

Fig. 4. The period frequency histograms for M 3 and M 15. Black bars show the contribution by fundamentalized first overtone pulsators.

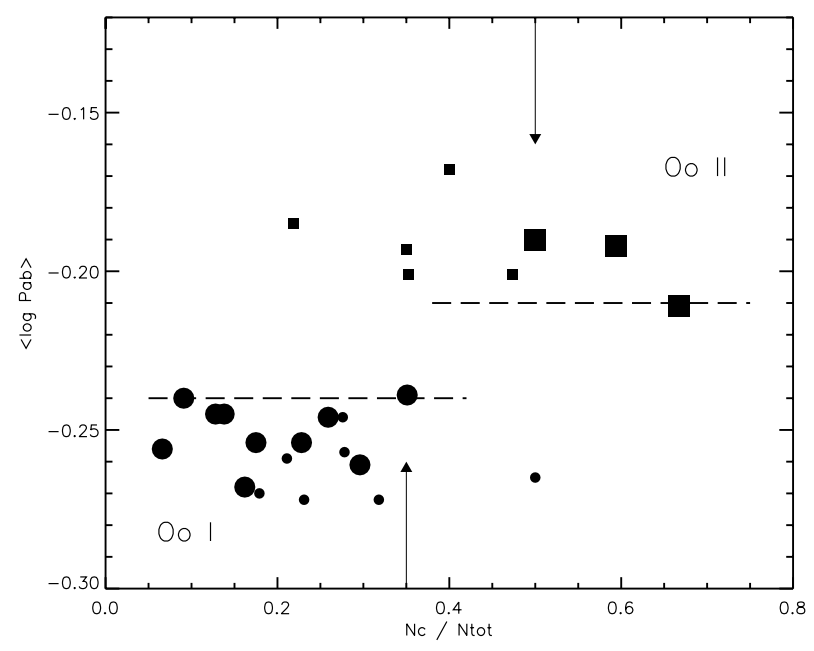

Fig. 5. The bimodal distribution of mean periods of RRab variables as a function of the number ratio of first overtone pulsator $\left(N_{\mathrm{c}}\right)$ to the total number of RR Lyrae $\left(N_{\mathrm{tot}}\right)$. Symbols as in previous figures.

periods at the larger metallicities. We conclude that the width in temperature of the instability strip appears largely independent of the cluster metallicity, whereas the evidence that the period distribution is not affected by the predicted variation of pulsators luminosity with metal abundance can be understood in terms of a correlated variation in the pulsator mass, as predicted by current evolutionary theories. 


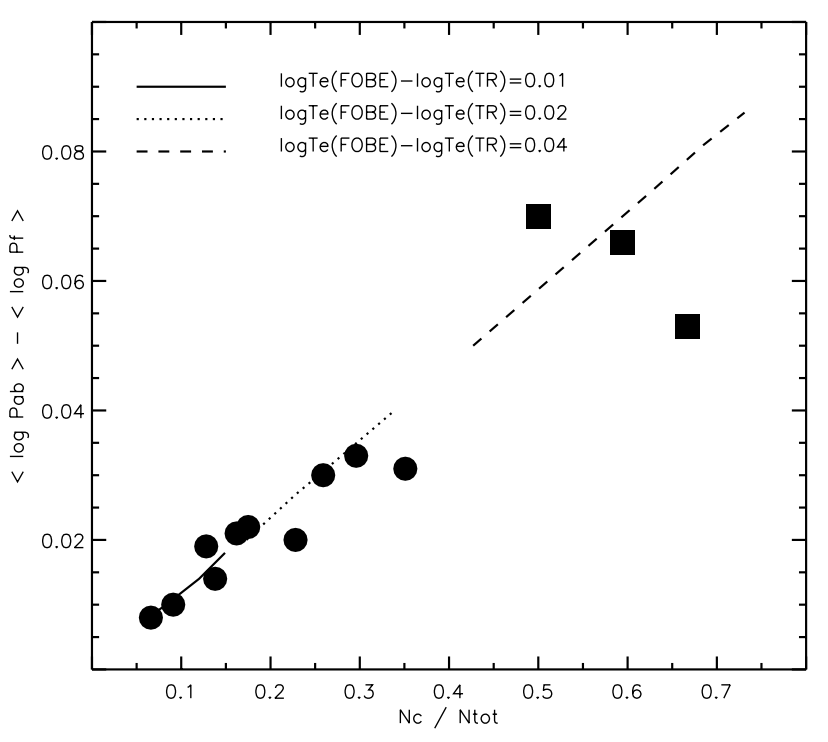

Fig. 6. The observed $<\log P_{\mathrm{ab}}>-<\log P f>$ for the most RR Lyrae rich globular clusters (symbols as in Fig. 2), as a function of the number ratio of first overtone pulsator $\left(N_{\mathrm{c}}\right)$ to the total number of RR Lyrae $\left(N_{\text {tot }}\right)$. The three segments show the theoretical predictions for the labelled assumption on the difference $\log T e(\mathrm{FOBE})-\log T e(\mathrm{TR})$ when varying HB morphology (see text).

We discuss mean periods, selecting from the sample of 32 clusters 26 clusters having no less than $10 \mathrm{RRab}$ and $5 \mathrm{RRc}$, but paying particular attention to clusters with more than 40 RR Lyrae stars, as the only ones with a sufficient statistical significance. One finds the well known Oosterhoff dichotomy in the mean $<P_{\mathrm{ab}}>$ periods, together with similarly clear evidence for a continuous behavior of the fundamentalized mean period $\langle P f\rangle$ in passing from OoII to OoI clusters, as well as for its constancy between the two groups, at fixed metal abundance.

On this basis the origin of the Oosterhoff dichotomy is briefly discussed, presenting evidences against a strong dependence of the HB luminosity on the metal content, as originally suggested by Sandage (1982) and more recently by D'Antona et al. (1997). On the contrary, the continuity of fundamentalized period, the comparison of the period frequency histograms in M 3 and M 15, the bimodal distribution of the relative abundance of first overtone pulsators and, last but not least, the observed differences between mean fundamental and fundamentalized periods, all agree in indicating the dominant occurrence of a variation in the pulsation mode, with variables in the OR zone pulsating in the fundamental or in the first overtone mode in OoI and OoII type clusters, respectively.
Acknowledgements. We are indebted to our anonymous referee for pertinent comments and suggestions that improved the first version of the paper. We gratefully acknowledge G. Bono, R. F. Butler, H. Smith and A. R. Walker for helpful suggestions. Financial support for this work was provided by the Ministero dell'Istruzione, dell'Università e della Ricerca (MIUR) under the scientific project "Stellar observables of cosmological relevance" (V. Castellani \& A. Tornambè, coordinators).

\section{References}

Bono, G., Caputo, F., Castellani, V., \& Marconi, M. 1997, A\&AS, 121, 327

Bono, G., Caputo, F., Castellani, V., \& Marconi, M. 2000, ApJ, 448, L115

Buonanno, R., Corsi, C. E., Fusi Pecci, F., Richer, H. B., \& Fahlman, G. G. 1993, AJ, 105, 184

Butler, R. F., Shearer, A., Redfern, R. M., et al. 1998, MNRAS, 296, 379

Carretta, E., \& Gratton, R. 1997, A\&AS, 121, 95

Caputo, F., de Stefanis, P., Paez, E., \& Quarta, M. L. 1987, A\&AS, 68, 119

Caputo, F., de Rinaldis, A., Manteiga, M., Pulone, L., \& Quarta, M. L. 1993, A\&A, 276, 41

Caputo, F., Castellani, V., Marconi, M., \& Ripepi, V. 1999, MNRS, 306,815

Caputo, F., Castellani, V., Marconi, M., \& Ripepi, V. 2000, MNRS, 316,819

Cassisi, S., Castellani, V., Degl'Innocenti, S., Salaris, M., \& Weiss, A. 1999, A\&AS, 134, 103

Castellani, V., \& Quarta, M. L. 1987, A\&AS, 71, 1

Clement, C. M., Muzzin, A., Dufton, Q., et al. 2001, AJ, 122, 2587

D’Antona, F., Caloi, V., \& Mazzitelli, I. 1997, ApJ, 477, 519

Demarque, P., Zinn, R., Lee, Y.-W., \& Yi, S. 2001, AJ, 119, 1398

Harris, W. E. 1996, AJ, 112, 1487

Lee, Y.-W. 1990, ApJ, 363, 159

Lee, Y.-W., Demarque, P., \& Zinn, R. 1990, ApJ, 350, 155

Lee, Y.-W., Demarque, P., \& Zinn, R. 1994, ApJ, 423, 248

Oosterhoff, P. Th. 1939, Observatory, 62, 104

Marconi, M., Caputo, F., Di Criscienzo, M., \& Castellani, M. 2003, ApJ, in press (Paper II)

Pritzl, B. J., Smith, H. A., Catelan, M., \& Sweigart, A. V. 2002, AJ, 124, 949

Rich, R. M., Sosin, C., Djorgovski, S. G., et al. 1997, ApJ, 484, 25

Rood, R. T. 1973, ApJ, 184, 815

Sandage, A. 1982, ApJ, 252, 553

Stellingwerf, R. F. 1975, ApJ, 195, 441

Stobie, R. S. 1971, ApJ, 168, 381

Sweigart, A. V., \& Catelan, M. 1998, ApJ, 501, L63

van Albada, T. S., \& Baker, N. 1973, ApJ, 185, 447 